\title{
Microdialysis Sampling of Renal Interstitial Fluid in Acute Studies
}

\author{
Wael Alanazi ${ }^{1}$, Selim Fakhruddin ${ }^{1} \&$ Keith E. Jackson ${ }^{1}$ \\ ${ }^{1}$ ULM School of Pharmacy, United States \\ Correspondence: Keith E. ULM School of Pharmacy, United States. E-mail: kjackson@ulm.edu
}

\author{
Received: September 24, 2015 Accepted: October 5, 2015 Online Published: December 16, 2015 \\ doi:10.5539/ijb.v8n1p69 \\ URL: http://dx.doi.org/10.5539/ijb.v8n1p69
}

\begin{abstract}
The microdialysis technique has been applied extensively during the last three decades where most of the research has focused primarily on the site of action. The microdialysis probe was developed for collection of interstitial fluid as well as delivering drugs to the target site. In comparison with other methods, the microdialysis technique has delivered more accurate results with minimal invasive procedures. Initially, the microdialysis technique was designed to quantitate dopamine levels in cerebral tissues, which facilitated the applications of this technology as a sampling tool. Presently the microdialysis technique has been applied to various organs such as the heart, kidney, and liver. In the current report, we describe the principle of the microdialysis technique and its application in in-vivo studies. Specifically, the present review provides the adaptation of this method in renal acute studies. However, the rationale for this report is to demonstrate that the microdialysis technique can be used to collect renal interstitial fluid in real time with no effect on the hemodynamic function.
\end{abstract}

Keywords: microdialysis, kidney, Angiotensin II, PGE2, interstitial fluid

\section{Introduction}

The importance of ISF sampling is its similarity to plasma in that it is the primary component of the extracellular fluid, and comprised of interstitial water, proteins, and other solutes. In 1972, based on the dialysis principle, Delgado succeeded to design dialytrode (a push-pull cannula connected with polysulfone membrane) for cerebral ISF collection in monkeys (Delgado, Lico, Bracchitta, \& Snyder, 1971). The disadvantages of push-pull techniques were overcome by Ungerstedt and Pycock. They employed a microdialysis probe that had a hollow fiber for continuous monitoring of dopamine levels in cerebral ISF (Ungerstedt, \& Pycock, 1974). During the last thirty years, numerous studies have shown the importance of the microdialysis technique in collecting cerebral ISF.

Advances over the years in microdialysis probe construction provide an advantage over the push-pull technique, where microdialysis probes are now less invasive, and contain semipermeable membranes, which allow for collection of a wide range of proteins. Moreover, microdialysis probes can be adapted to independently evaluate the concentration of different proteins based on their physicochemical properties like voltammetric techniques (Chefer, Thompson, Zapata, \& Shippenberg, 2009). Microdialysis is a powerful tool that can continuously evaluate neurotransmitter levels in the brain such as dopamine, norepinephrine, and serotonin (Hoebel, Hernandez, Schwartz, Mark, \& Hunter, 1989; Camp, DeJonghe, \& Robinson, 1997; Chaurasia, 1999). In addition, comparison of interstitial and plasma samples have shown significant differences in circulating hormone levels as compared to local tissue production. Thus, microdialysis produces a more accurate assessment of real-time solute changes in comparison to other sampling techniques.

\section{Microdialysis Technique}

\subsection{Principle of Microdialysis}

Microdialysis is a perfusion technique often used to collect ISF based on the dialysis principle. Insertion of a probe, which has a semipermeable membrane, allows solutes to move down their concentration gradients in a bidirectional manner. Thus, microdialysis sampling could be used for collecting various solutes, and for the determination of parent drug and drug metabolite concentrations in the cellular microenvironment. The microdialysis probe is designed to allow a particular size of solutes to pass through the micro-holes of a non-porous membrane. The restricted size of these holes prevents the intervention of macromolecules through the membrane, thus an analysis ready clear interstitial sample is collected (Figure 1). During the collection process, the probe is connected to a syringe pump that infuses perfusate at a constant rate. It is generally recommended that the 
infusion rate should be in a range between 0.5 to $5 \mu \mathrm{l} / \mathrm{min}$ (Chaurasia, 1999). This infusion range is slow enough to allow for bidirectional solute diffusion across the semipermeable membrane.

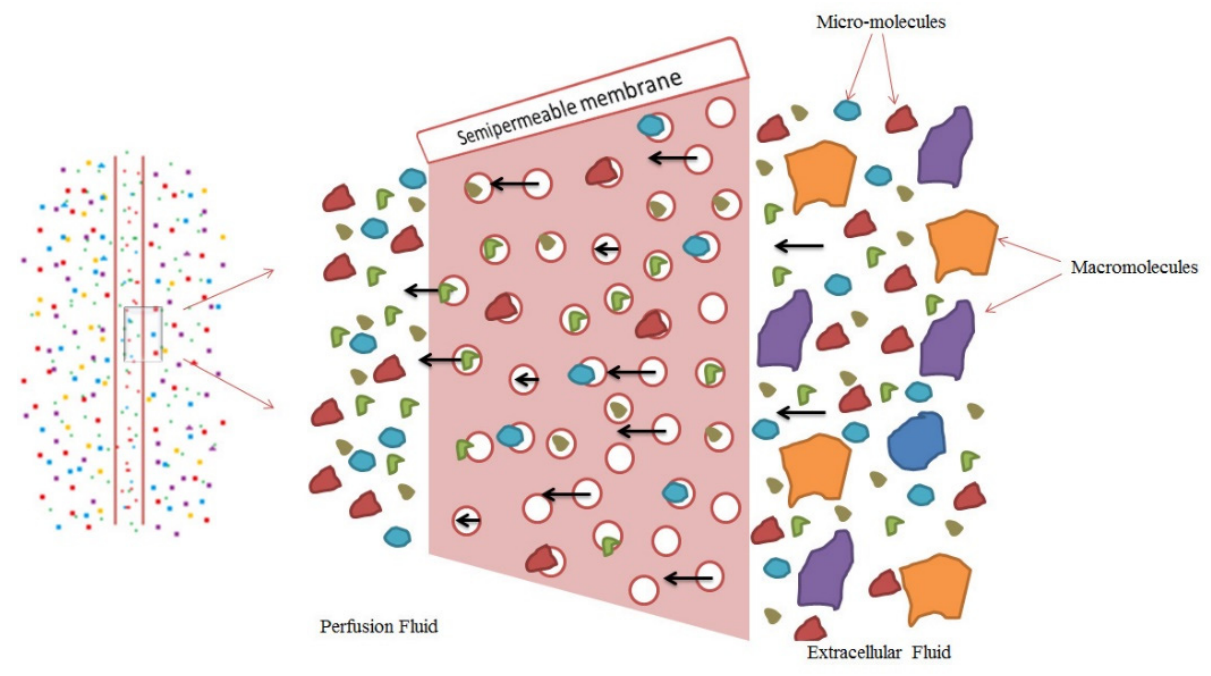

Figure 1. The diffusion process in the microdialysis probe

This figure shows the diffusion mechanism of micro-molecules through the probe's semipermeable membrane. At the same time, the macromolecules are entirely separated and excluded from collection with the perfusate.

The microdialysis probe is a concentric tube, in which the samples are carried out by the fluid flow. In between the inner tube and outer membrane, perfusion fluid and ISF pass through a semipermeable membrane. This layer is made of certain materials to achieve equilibrium at the site of fluid exchange between the perfusion liquid and ISF, where the filtrated fluid contains the target molecules (Lonnroth, Jansson, \& Smith, 1987). Polyarylethersulfone (Frost, Keen, Levine, \& Terasawa, 2008), polyethersulfone, polycarbonate, and regenerated cellulose (Hsiao, Ball, Morrison, Mefford, \& Bungay, 1990) are mainly utilized in manufacturing the semipermeable membranes for in-vitro and in-vivo studies. Recently, gelatin nanoparticles and chitosan were used to form a nanoporous chitosan membrane, which was applied in in-vitro experiments as a microdialysis technique (Chuang, M.Chen, \& P.Chen, 2014). In the manufacturing process, the goal of microdiaylsis membrane construction is to make a low molecular weight cutoff, which permits bidirectional diffusion of micro-molecules with no loss or gain of media or body fluids (Chaurasia et al., 2007). In order to deliver a particular drug or obtain interstitial samples, the probe membrane should be chosen with the proper cutoff to permit the diffusion of the targeted molecules (Chaurasia, 1999).

\subsection{Microdialysis Applications in in-vivo Study}

Microdialysis probes are designed in different sizes and shapes to facilitate ISF sampling and drug delivery in various organs and tissues. Probes with rigid cannulas are usually used for cerebral microdialysis, flexible and linear probes work well for peripheral microdialysis sampling. The selection of the probe and the microdialysis system is based on many factors for instance the site of interest (Kidney, heart or brain), the type of study (acute or chronic), and the animal condition (unconscious or freely moving animals).

Cerebral microdialysis research paved the way in the application of the microdialysis technique as a sampling and delivery method. This technique has the ability to reach the extracellular fluid of various intact tissues including the heart (Van Wylen et al., 1990; Kuzmin et al., 1992), kidney (Siragy, Jaffa, \& Margollius, 1993), liver (Scott, Bell, \& Lunte, 1989), and muscles (Lehmann, 1989). Van Wylen adapted it as a sampling tool to measure and monitor adenosine levels in regional cardiac ISF (Van Wylen et al., 1990). Then, Kuzmin succeeded in measuring extracellular adenine nucleotide breakdown products in intramyocardial ISF of canines (Kuzmin et al., 1992). During the last twenty years, cardiac microdialysis has facilitated the evaluation of the paracrine environment in different heart regions through placement of a less invasive probe.

Renal microdialysis has been decidedly employed in cortical and medullary renal research. Several studies have shown that the level of AngII is much higher in renal tissues than in plasma (Nishiyama, Seth, \& Navar, 2002; Siragy, Ibrahim, Jaffa, Mayfield, \& Margolius, 1994; Siragy, Howell, Ragsdale, \& Carey, 1995; Siragy, \& Carey, 1996; Siragy, \& Carey, 1997). Hence, evaluation of renal ISF in experimental models provides a better estimate of 
circulating AngII than plasma. Proceeding from that, Siragy developed and used renal microdialysis as a tool to evaluate the physiological and pathophysiological roles of AngII in the kidney (Siragy, Johns, Peach, \& Carey 1992; Siragy et al., 1993). Renal microdialysis was later developed to evaluate cortical ISF for AngII and ATP over a 14 day period in a chronic hypoglycemic animal model (Prathipati, Alanazi, Fakhruddin, D. Jackson, \& K. Jackson, 2015). In addition, the microdialysis technique was applied in humans by inserting a semipermeable membrane needle into the upper trapezius muscle to obtain clear muscular ISF samples (Shah, Phillips, Danoff, \& Gerber, 2005). In comparison to other in vivo sampling techniques, microdialysis provides the advantage of simultaneous sampling at multiple target sites.

Thus, the microdialysis technique is a reliable tool in acute and chronic studies to measure the concentration of the targeted molecules in ISF of various organs (Figure 2).

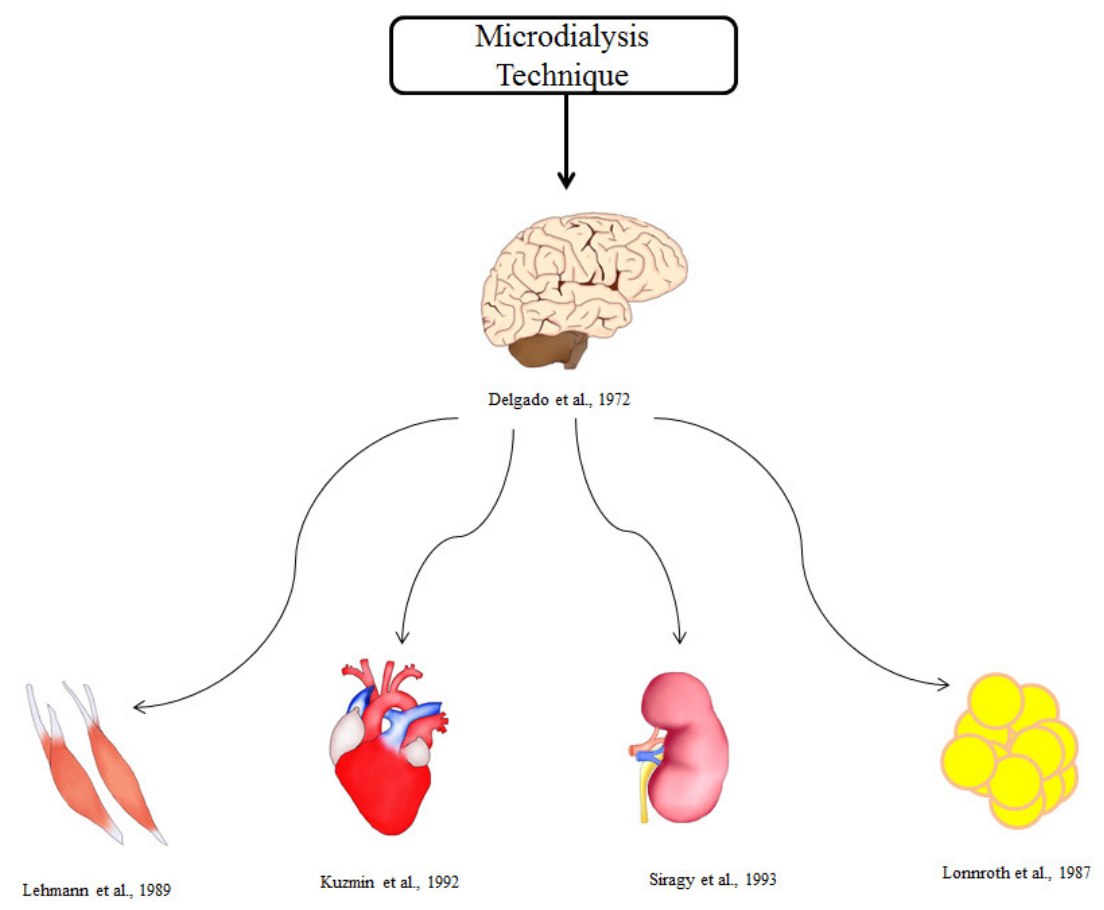

Figure 2. In vivo microdialysis applications in various organs

The cerebral microdialysis technique has been developed to be used in different targets such as the muscular, cardiac, renal, and adipose tissues.

\section{Adaptation of Microdialysis Technique in Acute Renal Studies}

Angiotensin II (AngII) controls several autocrine and paracrine signaling pathways in both physiological and pathophysiological conditions, thus AngII is known as a potent effector peptide in renal tissues. Therefore, it is important to investigate the modulatory or inhibitory effects of AngII on various biological agents such as prostaglandins (PGs), and interleukins (ILs). It has been standard practice in many reports to evaluate the role of AngII in the kidneys via assessing all interesting molecules in serum and urine. Recently, a new strategy has been designed that involves inspecting the changes in renal molecules through direct sampling of the renal interstitial fluid (ISF). This strategy produced more valuable results since most of the interesting molecules are mainly located within the tissues. Hence, the best way to assess the local effect of AngII and other peptides is by collecting the interstitial fluid that surrounds the cells in the site of interest.

The renin angiotensin aldosterone system (RAS) exerts a critical role in regulating blood pressure and body fluid hemostasis. This system modulates various autocrine and paracrine signaling pathways in tissues and organs. The primary effector molecule of RAS is AngII. AngII is a systemic vasoconstrictor and reactive oxygen species (ROS) mediator. AngII has emerged as a critical hormone that affects the function of virtually all organs, including heart, kidney, vasculature, and brain, where it has both beneficial and pathological effects. AngII formation begins as angiotensinogen secretion in the bloodstream. Angiotensinogen is cleaved by renin to produce angiotensin I (AngI), which is the precursor of AngII. AngI is converted to AngII in the presence of angiotensin-converting enzyme 2 (ACE2) that is released by various tissues. In kidneys, AngII has numerous physiological functions 
including vasoconstriction of the renal arterioles and induction of sodium/water reuptake to maintain renal blood flow and glomerular filtration rate (GFR).

AngII type 1 receptors (AT1) promote hypertension by inducing renal and systemic vasoconstriction and by decreasing sodium/water excretion. In the renal microvasculature, prostaglandins (PGs) mainly PGE2 counteracts the vasoconstrictive actions of AngII and regulates water/ions reabsorption (Inscho, Carmines, \& Navar, 1990). Thus, PGE2 promotes an antihypertensive effect in the face of AngII mediated elevations in blood pressure.

PGs are involved in many physiological and pathophysiological functions for instance platelet aggregation, inflammation, fever, vasoconstriction, and vasodilation (Bos, Richel, Ritsema, Peppelenbosch, \& Versteeg, 2004; Inscho et al., 1990). PGs are metabolites of arachidonic acid, and they are formed in response to a variety of stimuli such as AngII and growth factors (Jaimes, Tian, Pearse, \& Raij, 2005; Goppelt-Struebe, Stroebel, \& Hoppe, 1996). The first step in prostanoids formation (prostaglandins and thromboxanes) is arachidonic acid release from the phospholipid cell membrane through the interaction with a phospholipase A2 enzyme. Then, arachidonic acid is converted by COX1 and COX2 to PGH2, the precursor of all prostanoids (PGE2, PGI2, PGD2, PGF2 $\alpha$, and TXA2) (Zarghi \& Arfaei, 2011; Bos et al., 2004). AngII activates prostanoids formation through two mechanisms; namely by inducing PLA2 activity and COX2 production at the mRNA and protein levels (Hu, Kerb, Shi, Wei-Lavery, \& Hoffman, 2002). Specifically, in the renal tissues, enhancing PGE2 production during AT1 receptor activation counteracts AngII-induced vasoconstriction in the renal microcirculation (Imig, 2006).

The kidney has gain increased attention over the past years as an interesting target to evaluate the role of various molecules. As previously mentioned, the renal concentrations of targeted substances are often different from their plasma levels. For instance, some reports have demonstrated that intra-renal AngII concentrations are 1000 fold higher than circulating plasma concentrations (Campbell, Lawrence, Towrie, Kladis, \& Valentijn, 1991). This results from a high expression of AT1, which promotes an elevation in AngII uptake and induces angiotensinogen mRNA expression (Navar, Prieto, Satou, \& Kobori, 2011). In addition, Navar et al. have demonstrated in a previous report that the entire RAS is present within the renal tissue (Navar et al., 2011). Thus, current investigations on the role of the RAS in hypertension and kidney diseases have changed focus to study local production in a particular tissue such as the kidney, and heart verses evaluating global alterations of the RAS. Consequently, it was preferred to monitor the content of RAS and its intra-renal function locally by collecting and evaluating renal or cardiac ISF samples. By using the microdialysis technique, renal ISF was sampled to determine the intra-renal AngI and AngII concentrations in the renal cortex, and compared with their plasma concentrations (Nishiyama et al., 2002).

Previous reports have described in detail the surgical methods of renal microdialysis probe implantation (Siragy et al., 1992; Siragy et al., 1995). In the current report, the surgical protocol, and blood vessel cannulation are described (Figure 3). Additionally, implantation of the microdialysis probe into the renal cortex and the diffusion mechanism of the targeted molecules through the probe are shown (Figure 4). All in all, renal microdialysis sampling during acute hemodynamic studies is presented in this report (Figure 5). Based on this methodology, many studies have successfully collected ISF and evaluated local alterations in targeted substances during various experimental conditions.
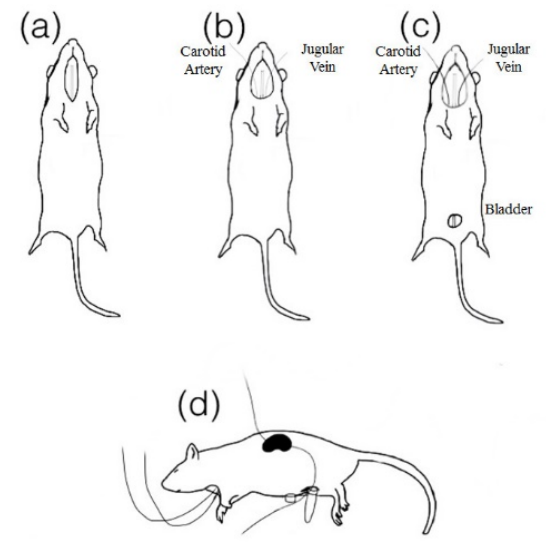

Figure 3. Surgical steps for renal microdialysis in acute study

Sketch of surgical procedures involved in acute renal microdialysis probe implantation. (a) Tracheal cannulation. (b) Carotid artery and Jugular vein cannulations. (c) Bladder catheterization. (d) Probe insertion in renal cortex for collecting ISF samples. 

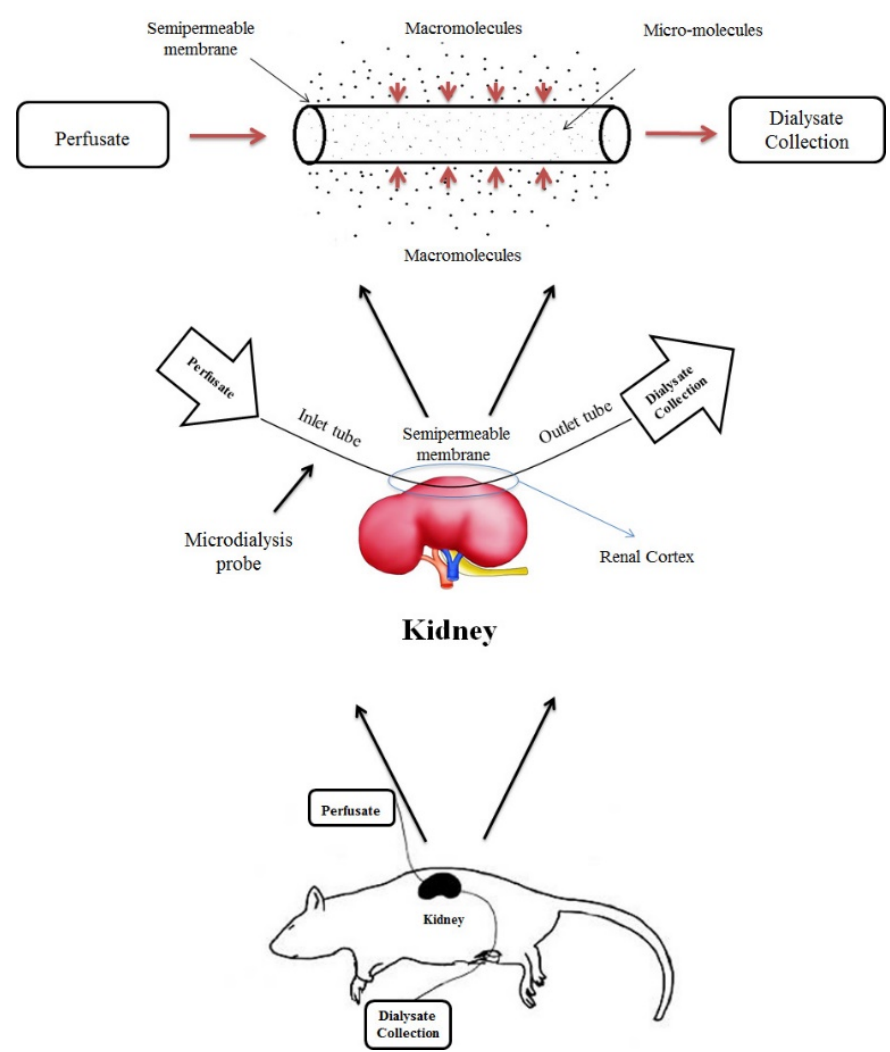

Figure 4. The mechanism of microdialysis sampling in renal cortex

This figure shows how the probe collects ISF that contains the target molecules in cortical tissues. During perfusate infusion, solutes in the renal cortex are diffused through the semipermeable membrane and collected with the dialysate.

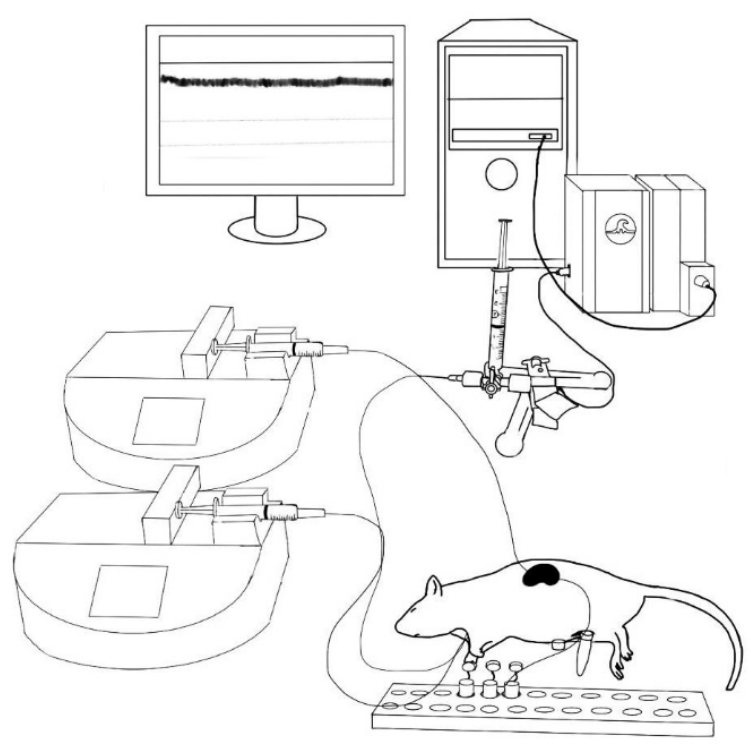

Figure 5. The protocol of renal microdialysis in acute study

This figure shows a pictorial representation of an acute renal microdialysis experiment. The carotid artery is connected by polyethylene tubing to a pressure transducer which is coupled with a polygraph system and a personal computer to measure the blood pressure, and heart rate. The jugular vein and the inlet tube of the microdialysis probe are connected to microinfusion pumps to infuse the treatment intravenously, and collect renal ISF samples, respectively. The microdialysis samples are collected in Eppendorf tubes from the outlet tube. 
By using the microdialysis technique, Siragy et al. have studied and published many reports on the ability of AngII to alter local renal production of various molecules such as PGs, bradykinin, and ATP. During intravenous infusion of AngII, the renal changes in the targeted substances have been identified by analyzing the collected renal ISF. Several reports have noted that the microdialysis probe should be inserted into the renal cortex to avoid disruption of normal renal function. One of the essential targets in the renal tissues is PGs, where AngII augments the formation of PGE2 to maintain normal renal microcirculation blood flow. Recently, four hours of low dose AngII $(7 \mathrm{ng} / \mathrm{kg} / \mathrm{min})$ infusion promoted an induction in PGE2 levels that were monitored by inserting a linear microdialysis probe into the renal cortex (Alanazi, Fakhruddin \& Jackson, 2015 unpublished) (Figure 6 and 7). Normal renal function was observed during the entirety of the 4 hour study and no perceived alterations in hemodynamic function were noted.

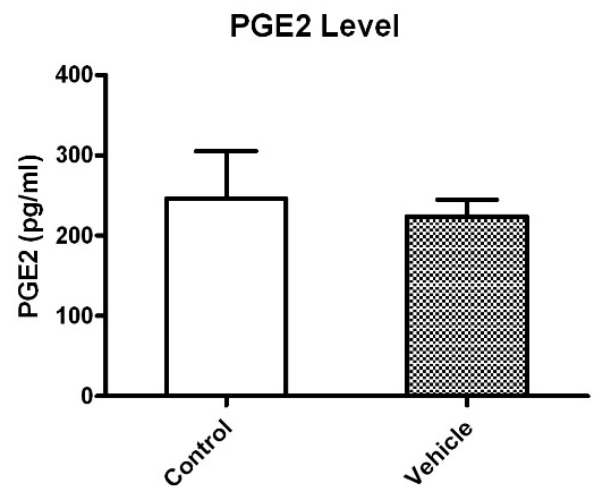

Figure 6. Analysis of PGE2 concentration in renal ISF during vehicle infusion

In acute renal studies, there were no significant changes in renal cortical PGE2 levels during vehicle infusion as compared to control.

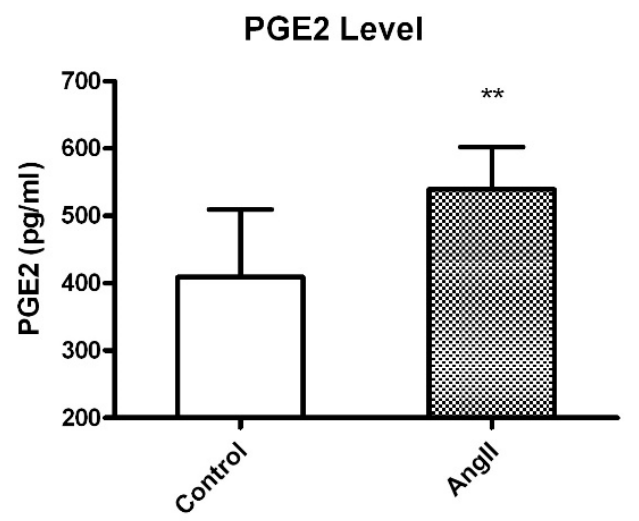

Figure 7. Analysis of PGE2 concentration in renal ISF during AngII infusion

Acute AngII infusion produced a significant increase in renal ISF PGE2 levels as compared to control.

\section{Systemic Effects of the Inserted Microdialysis Probe}

The idea of inserting the microdialysis probe in the tissue is to collect ISF with minimal passive effects on blood circulation and target organ function. Some reports in brain research have shown the microdialysis technique as an invasive sampling tool which caused tissue perturbation. Hence, it was concluded that microdialysis implantation leads to severe damage in the blood-brain barrier (Allen, Crooks, \& Yokel, 1992). Currently, there have been many technological advances in microdialysis probe design and variety, which has alleviated many of the previous drawbacks. Recent research has shown adaptation of neural microdialysis probes in exercise research, where the neural ISF was collected in a moving animal with nominal tissue injury (Meeusen, Piacentini, \& De Meirleir, 2001). Also, cardiac microdialysis probes have been an effective collecting tool with no disturbances in heart 
function as well as in sympathetic and parasympathetic signals. As previously described, the probe was placed into the cardiac sinoatrial node with no irregular neuronal inputs (Jackson, Farias, \& Caffrey, 2000). In acute anesthetized animal studies, renal microdialysis facilitated ISF sampling, during hemodynamic function monitoring. In addition, probe insertion in renal tissues has no effect on systemic hemodynamic function and heart rate during acute studies (Alanazi et al., 2015 unpublished) (Figures 8 and 9).

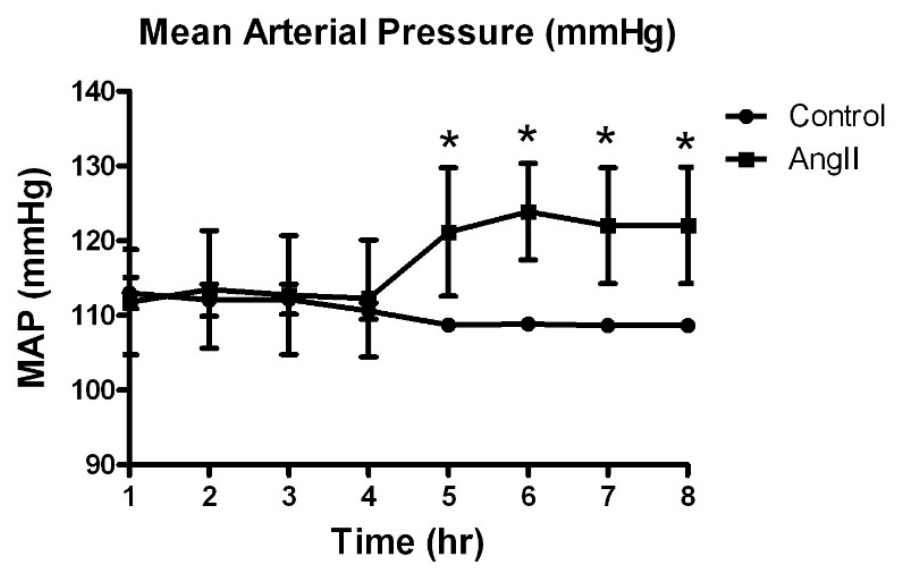

Figure 8. Mean arterial pressure measurement

This figure shows MAP response for acute AngII infusion. AngII increased MAP significantly as compared to control. There were no significant differences in control blood pressure due to probe insertion.

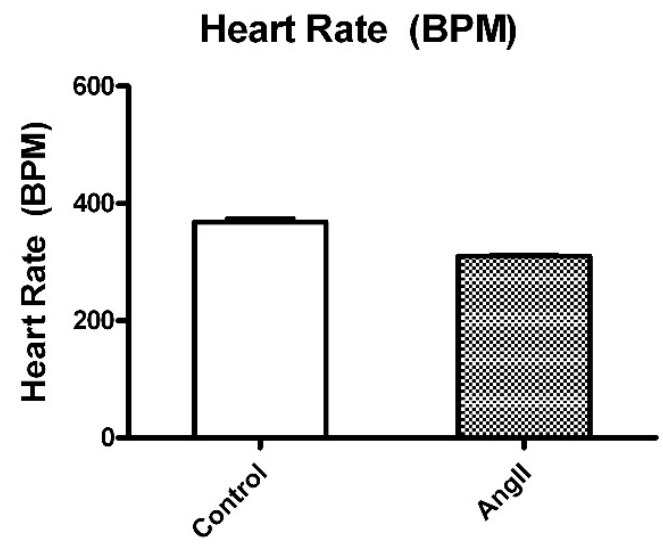

Figure 9. Heart rate measurement

There were no significant differences in heart rate during acute AngII infusion. Probe insertion produced no significant changes in heart rate in control or AngII infused animals.

\section{Approaches for Interstitial Fluid Analysis}

During the collection process, the infusion rate of the fluid is usually slow; therefore the volume of collected samples is typically small. Sample size also depends on the duration of the control and treatment periods. Many approaches have been used to analyze the microdialysis samples. They can be categorized into two main approaches, the connected analytical approach, and the external analytical approach. During the connected analytical approach, facilitates immediate analysis, since samples flow through an on-line device, such as capillary electrophoresis (Phillips, Kennedy, \& DeFabo, 1997), and High-performance liquid chromatography (HPLC) (Chaurasia, Chen, \& Ashby, 1999). On the other hand, the external or non-connected analytical approach allows the collected samples to be stored until analyzed by radioimmunoassay (RIA) or enzyme-linked immunosorbent assay (ELISA) (Maidment, Brumbaugh, Rudolph, Erdelyi, \& Evans, 1989). In general, the selected approach for ISF analysis should be sensitive and accurate enough to detect the molecules of interest from small sample amounts. 


\section{Advantages of using Microdialysis Technique in Acute Studies}

The advantage of using microdiaysis in acute studies is that it allows for less invasive surgical procedures and facilitates evaluation of systemic hemodynamic function without disturbances in blood pressure and heart rate. Currently, probes can be easily placed into the kidney through a small incision in the back. However, midline laparotomy which was previously employed represents a more intrusive technique as shown in previous brain research studies. After probe implantation, the flexibility of the microdialysis probe allows the exposed kidney to be returned smoothly to the abdominal cavity, thus avoiding the traditional open abdominal surgery. Furthermore, previous studies have found that the implantation of smaller diameter probes with less than 24 hours under study produces less harm to the target tissue. Following the stabilization period, ISF can be harvested before, during, and after treatment based on set time frames (Figure 10). Consequently, a single animal could be used as its own control, thus reducing inter-animal variability (Meeusen, Piacentini, \& De Meirleir, 2001). In addition, several endogenous molecules can be monitored in different organs of the same animal, during selected control and treatment periods. In general, the current probe insertion methods have no noted effects on renal and systemic hemodynamic function due to avoidance of major surgical protocols, and minimal blood loss. On the other hand, traditional arterial blood sampling might cause disturbances in blood pressure during acute studies specifically in hemodynamic studies, where the repeating collection leads to inaccurate results due to the changes in hemodynamic functions and excessive blood loss in extended time acute studies. Blood collection is used to obtain plasma samples that evaluate only the systemic changes in the substance of interest, where blood collection is still unable to measure the local changes in a particular tissue or organ. In addition, acute microdialysis studies have demonstrated that significant renal interstitial changes in the targeted molecules occur during systemic infusion of different agents such as AngII or RAS inhibitors (Siragy et al., 1993), (Alanazi et al., 2015 unpublished). Infusion of AngII promoted a significant increase in renal cortical PGs levels obtained via microdialysis (Siragy et al., 1993), (Alanazi et al., 2015 unpublished). The observed increase in PGs levels was attenuated by administration of an AT1 receptor antagonist (Siragy et al., 1993).

Over time microdialysis has evolved as a sensitive technique to provide a good evaluation of local solute changes in the site of interest. Additionally, microdialysis can be used to administer drugs directly to target tissues, thus avoiding unwanted adverse systemic reactions. For instance, initial clinical uses of microdialysis probes were to determine the unbound drug concentrations in the treated tissues, and to evaluate the pharmacokinetic properties of administered drugs. Microdialysis is widely used in antibiotics research to study the effectiveness of antimicrobial drugs and to determine drug resistance. Currently, microdialysis is used for various clinical purposes such as in topical drug administration to avoid adverse systemic effects as well as in cancer therapy to inhibit tumor growth and reduce cytotoxic actions on normal cells. In cancer research, microdialysis is used to evaluate the cytotoxic penetration features of anticancer drugs and to provide pharmacokinetics characteristics of anticancer drugs in an effort to improve their antineoplastic effects.

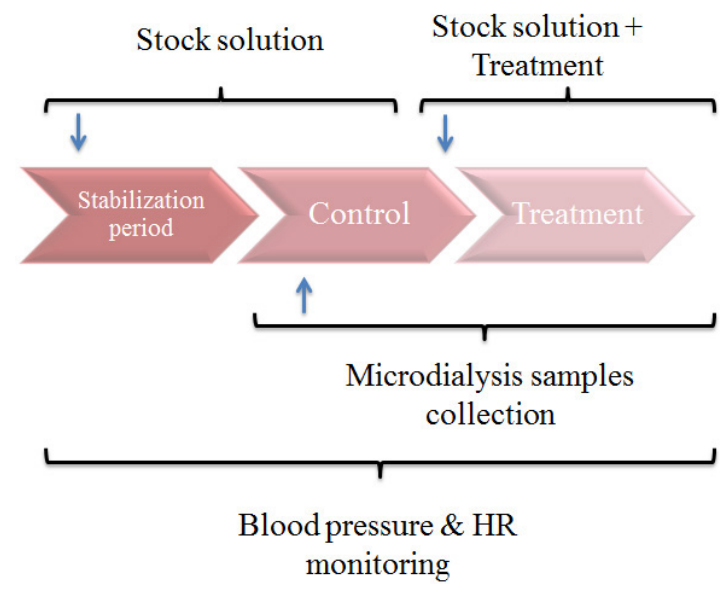

Figure 10. Renal microdialysis experimental protocol.

This flowchart shows the acute experimental protocol during renal microdialysis. After a stabilization period, microdialysis samples are collected during the control and treatment periods. 


\section{Conclusion}

Microdialysis is rapidly becoming the goal standard for ISF collection. The microdialysis technique has improved accuracy of determining endogenous compound and drug concentration levels as compared to only measuring their plasma concentrations. Initially, microdialysis probes were developed to investigate alterations in neurotransmitter levels in brain research. Current probes have been redesigned with different shapes and dimensions to monitor local changes in several organs, where they can be concurrently targeted in the same animal (Muller, 2002).

In vivo acute renal studies, which utilize microdialysis have been able to evaluate local changes in endogenous compound such as AngII, ATP, and PGs without significant alterations in hemodynamic function. In addition, ISF has been collected in pharmacokinetic-pharmacodynamic drug research to improve reliability of results between the drug response and its concentration in the tissue of interest. Therefore, drug investigation drawbacks present due to collecting plasma samples could be avoided by direct ISF harvesting. ISF samples do not require enzyme inhibitors or centrifugation processes since the microdialysis membrane prevents degradation of enzymes and diffusion of macromolecules into the collected samples. Hence, the obtained microdialysis samples can be directly analyzed or stored until analyzed.

Microdialysis has been adapted as a sampling method for accurate assessment of extracellular peptides and drug concentrations. This method is widely used in that it involves less invasive procedures, produces more reliable results, and promotes a significant reduction in animal numbers per study. Ultimately, the current applications of microdialysis will have a critical bearing on ISF sampling of various organs in future research.

\section{Acknowledgments}

This work was supported by a NIH-LBRN grant and a SACM fellowship.

\section{References}

Allen, D. D., Crooks, P. A., \& Yokel, R. A. (1992). 4-Trimethylammonium antipyrine: a quaternary ammonium nonradionuclide marker for blood-brain barrier integrity during in vivo microdialysis. $J$ Pharmacol Toxicol Methods, 28(3), 129-135. http://dx.doi.org/10.1016/1056-8719(92)90074-B

Bos, C. L., Richel, D. J., Ritsema, T., Peppelenbosch, M. P., \& Versteeg, H. H. (2004). Prostanoids and prostanoid receptors in signal transduction. The International Journal of Biochemistry \& Cell Biology, 36(7), 1187-1205. http://dx.doi.org/10.1016/j.biocel.2003.08.006

Camp, D. M., DeJonghe, D. K., \& Robinson, T. E. (1997). Time-dependent effects of repeated amphetamine treatment on norepinephrine in the hypothalamus and hippocampus assessed with in vivo microdialysis. Neuropsychopharmacology, 17(3), 130-140. http://dx.doi.org/10.1016/S0893-133X(97)00035-3

Campbell, D. J., Lawrence, A. C., Towrie, A., Kladis, A., \& Valentijn, A. J. (1991). Differential regulation of angiotensin peptide levels in plasma and kidney of the rat. Hypertension, 18(6), 763-773. http://dx.doi.org/10.1161/01.HYP.18.6.763

Chaurasia, C. S. (1999). In vivo microdialysis sampling: theory and applications. Biomed Chromatogr, 13(5), 317-332. http://dx.doi.org/10.1002/(SICI)1099-0801(199908)13:5\%3C317::AID-BMC891\%3E3.0.CO;2-I

Chaurasia, C. S., Chen, C. E., \& Ashby, C. R. (1999). In vivo on-line HPLC-microdialysis: simultaneous detection of monoamines and their metabolites in awake freely-moving rats. J Pharm Biomed Anal., 19(3-4), 413-422. http://dx.doi.org/10.1016/S0731-7085(98)00182-4

Chaurasia, C. S., Muller, M., Bashaw, E. D., Benfeldt, E., Bolinder, J., Bullock, R., ... Yeo, H. (2007). AAPS-FDA workshop white paper: microdialysis principles, application and regulatory perspectives. Pharm Res., 24(5), 1014-1025. http://dx.doi.org/10.1007/S11095-006-9206-Z

Chefer, V. I., Thompson, A. C., Zapata, A., \& Shippenberg, T. S. (2009). Overview of Brain Microdialysis. Curr Protoc Neurosci., 47(7.1), 1-7. http://dx.doi.org/10.1002/0471142301.ns0701s47

Chuang, Y. J., Mei-Jung Chen, M. J., \& Chen, P. R. (2014). Fabrication and Permeability Characteristics of Microdialysis Probe Using Chitosan Nanoporous Membrane. Journal of Nanomaterials, 2014, Article ID 968098, 11 pages. http://dx.doi.org/10.1155/2014/968098

Delgado, J. M., Lico, M. C., Bracchitta, H., \& Snyder, D. R. (1971). Brain excitability and behavioral reactivity in monkeys under meprobamate. Arch Int Pharmacodyn Ther., 194(1), 5-17. 
Frost, S. I., Keen K. L., Levine, J. E., \& Terasawa, E. (2008). Microdialysis methods for in vivo neuropeptide measurement in the Stalk-median eminence in the Rhesus monkey. Journal of neuroscience methods, 168(1), 26-34. http://dx.doi.org/10.1016/j.jneumeth.2007.09.001

Goppelt-Struebe, M., Stroebel, M., \& Hoppe, J. (1996). Regulation of platelet-derived growth factor isoform-mediated expression of prostaglandin G/H synthase in mesangial cells. Kidney Int., 50(1), 71-78. http://dx.doi.org/10.1038/ki.1996.288

Hoebel, B. G., Hernandez, L., Schwartz, D. H., Mark, G. P., \& Hunter, G. A. (1989). Microdialysis studies of brain norepinephrine, serotonin, and dopamine release during ingestive behavior. Theoretical and clinical implications. Annals of the New York Academy of Sciences, 575(1), 171-193. http://dx.doi.org/10.1111/j. 1749-6632.1989.tb53242.X

Hsiao, J. K., Ball, B. A., Morrison, P. F., Mefford, I. N., \& Bungay, P. M. (1990). Effects of different semipermeable membranes on in vitro and in vivo performance of microdialysis probes. J. Neurochem, 54(4), 1449-1452. http://dx.doi.org/10.1111/J.1471-4159.1990.TB01982.X

Hu, Z. W., Kerb, R., Shi, X. Y., Wei-Lavery, T., \& Hoffman, B. B. (2002). Angiotensin II increases expression of cyclooxygenase-2: implications for the function of vascular smooth muscle cells. J Pharmacol Exp Ther., 303(2), 563-573. http://dx.doi.org/10.1124/jpet.102.037705

Imig, J. D. (2006). Eicosanoids and renal vascular function in diseases. Clin Sci., 111(1), 21-34. http://dx.doi.org/10.1042/CS20050251

Inscho, E. W., Carmines, P. K., \& Navar, L. G. (1990). Prostaglandin influences on afferent arteriolar responses to vasoconstrictor agonists. Am J Physiol., 259(1 Pt 2), F157-163.

Jackson, K., Farias, M., \& Caffrey J. L. (2000). Cardiac microdialysis a powerful tool. Cardiovasc Res., 46(3), 367-369. http://dx.doi.org/10.1016/S0008-6363(00)00085-7

Jaimes, E.A., Tian, R., X., Pearse, D., \& Raij L. (2005). Up-regulation of glomerular COX-2 by angiotensin II: role of reactive oxygen species. Kidney Int. 68(5), 2143-2153. http://dx.doi.org/10.1111/j.1523-1755.2005. 00670.x

Kuzmin, A. I., Tskitishvili, O. V., Serebryakova, L. I., Saprygina, T. V., Kapelko, V. I., \& Medvedev, O. S. (1992). Cardiac microdialysis measurement of extracellular adenine nucleotide breakdown products during regional ischemia and reperfusion in canine heart: protective effect of propranolol against reperfusion injury. Journal of Cardiovascular pharmacology, 20(6), 961-968. http://dx.doi.org/10.1097/00005344-199212000-00017

Lehmann, A. (1989). Effects of microdialysis-perfusion with anisoosmotic media on extracellular amino acids in the rat hippocampus and skeletal muscle. J. Neurochem, 53(2), 525-535. http://dx.doi.org/10.1111/j.1471-4159.1989.tb07365.x

Lonnroth, P., Jansson, P. A., \& Smith, U. (1987). A microdialysis method allowing characterization of intercellular water space in humans. Am. J. Physiol., 253(2 Pt 1), E228-231.

Maidment, N. T., Brumbaugh, D. R., Rudolph, V. D., Erdelyi, E., \& Evans, C. J. (1989). Microdialysis of extracellular endogenous opioid peptides from rat brain in vivo. Neuroscience, 33(3), 549-557. http://dx.doi.org/10.1016/0306-4522(89)90407-7

Meeusen, R., Piacentini M. F., \& De Meirleir K. (2001). Brain microdialysis in exercise research. Sports Med, 31(14), 965-983. http://dx.doi.org/10.2165/00007256-200131140-00002

Muller, M. (2002). Science, medicine, and the future: Microdialysis. BMJ, 324(7337), 588-591.

Navar, L. G., Prieto, M. C., Satou, R., \& Kobori, H. (2011). Intrarenal angiotensin II and its contribution to the genesis of chronic hypertension. Curr Opin Pharmacol, 11(2), 180-186. http://dx.doi.org/10.1016/j.coph. 2011.01.009

Nishiyama, A., Seth, D. M., \& Navar, L. G. (2002). Renal Interstitial Fluid Concentrations of Angiotensins I and II in Anesthetized Rats. Hypertension, 39(1), 129-134. http://dx.doi.org/10.1161/hy0102.100536

Phillips, T. M., Kennedy, L. M., \& DeFabo, E. C. (1997). Microdialysis-immunoaffinity capillary electrophoresis studies on neuropeptide-induced lymphocyte secretion. J. Chromatogr. B., 697(1-2), 101-109. http://dx.doi.org/10.1016/S0378-4347(97)00132-1 
Prathipati, P., Alanazi, W., Fakhruddin, Jackson, D. W., \& Jackson, K. E. (2015). Role of interstitial angiotensin II and ATP in mediating renal injury induced by recurrent insulin induced hypoglycemia. Annual Research \& Review in Biology, 6(5), 328-336. http://dx.doi.org/10.9734/ARRB/2015/16184

Scott, D. O., Bell, M. A., \& Lunte, C. E. (1989). Microdialysis-perfusion sampling for the investigation of phenol metabolism. J. Pharm. Biomed. Anal., 7(11), 1249-1259. http://dx.doi.org/10.1016/0731-7085(89)80128-1

Shah, J. P., Phillips, T. M., Danoff, J. V., \& Gerber, L. H. (2005). An in vivo microanalytical technique for measuring the local biochemical milieu of human skeletal muscle. J Appl Physiol, 99(5), 1977-1984. http://dx.doi.org/10.1152/japplphysiol.00419.2005

Siragy, H. M., \& Carey, R. M. (1996). The subtype-2 (AT2) angiotensin receptor regulates renal cyclic guanosine 3', 5'-monophosphate and AT1 receptor-mediated prostaglandin E2 production in conscious rats. J. Clin. Invest., 97(8), 1978-1982. http://dx.doi.org/10.1172/JCI118630

Siragy, H. M., \& Carey, R. M. (1997). The subtype 2 (AT2) angiotensin receptor mediates renal production of nitric oxide in conscious rats. J. Clin. Invest., 100(2), 264-269. http://dx.doi.org/10.1172/JCI119531

Siragy, H. M., Howell, N. L., Ragsdale, N. V., \& Carey, R. M. (1995). Renal interstitial fluid angiotensin. Modulation by anesthesia, epinephrine, sodium depletion, and renin inhibition. Hypertension, 25(5), 1021-1024. http://dx.doi.org/10.1161/01.HYP.25.5.1021

Siragy, H. M., Ibrahim, M. M., Jaffa, A. A., Mayfield, R., \& Margolius, H. S. (1994). Rat renal interstitial bradykinin, prostaglandin E2, and cyclic guanosine 3', 5'-monophosphate. Effects of altered sodium intake. Hypertension, 23(6 PT 2), 1068-1070. http://dx.doi.org/10.1161/01.HYP.23.6.1068

Siragy, H. M., Jaffa, A. A., \& Margollius, H. S. (1993). Stimulation of renal interstitial bradykinin by sodium depletion. Am. J. Hypertens, 6(10) 863-866.

Siragy, H. M., Johns, R. A., Peach, M. J., \& Carey, R. M. (1992). Nitric oxide alters renal function and guanosine 3',5'-cyclic monophosphate. Hypertension, 19 (6 Pt 2), 775-779. http://dx.doi.org/10.1161/01.HYP.19.6.775

Ungerstedt, U., \& Pycock, C. (1974). Functional correlates of dopamine neurotransmission. Bull Schweiz Akad Med Wiss, 30(1-3), 44-55.

Van Wylen, D. G., Willis, J., Sodhi, J., Weiss, R. J., Lasley, R. D., \& Mentzer, R. M. (1990). Cardiac microdialysis to estimate interstitial adenosine and coronary blood flow. Am. J. Physiol, 258(6), H1642-1649.

Zarghi, A., \& Arfaei, S. (2011). Selective COX-2 Inhibitors: A Review of Their Structure-Activity Relationships. Iranian Journal of Pharmaceutical Research, 10(4), 655-683.

\section{Copyrights}

Copyright for this article is retained by the author(s), with first publication rights granted to the journal.

This is an open-access article distributed under the terms and conditions of the Creative Commons Attribution license (http://creativecommons.org/licenses/by/3.0/). 